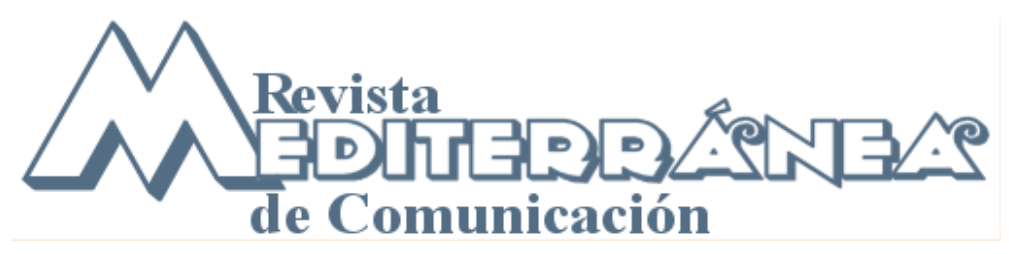

\title{
La investigación académica sobre periodismo en México: una mirada crítica
}

\section{The academic research on Journalism in Mexico: a critical view}

\author{
Prof. Dra. Montserrat Jurado Martín \\ Profesora asociada de la Universidad Miguel Hernández de Elche (España) \\ Profesora titular en el Centro de Estudios Ciudad de la Luz (España) \\ mjurado@umh.es, mjurado@ciudaddelaluz.es
}

\begin{abstract}
Resumen
El estudio de los medios de comunicación permite conocer el perfil de una sociedad a través de los temas que le son de interés y que vienen publicados de manera diaria en las páginas de los periódicos. Los contenidos que se reflejan en sus páginas integran la historia contemporánea más actual y dan una visión concreta y específica de ese contexto. A estos contenidos se suele llegar mediante un análisis de contenido de los mismos periódicos y en el caso de México este tipo de investigaciones son muy escasas. En este artículo se lleva a cabo un pormenorizado estudio que confirma esta afirmación y se indaga en las cuestiones que hacen que este tipo de estudio no sea recurrente para los investigadores. Las características más singulares en este trabajo basado en la búsqueda de las referencias bibliográficas que respalden la hipótesis y la metodología del análisis de caso en el estado de Colima (México) concluyen que falta bibliografía adecuada y actual, fuentes hemerográficas dignas, inversión e interés por los temas relacionados con el Periodismo y en general con las Ciencias de la Comunicación desde el punto de vista de los contenidos que se publican en prensa. El artículo propone también la necesidad de la inversión en investigación, su posterior difusión y la concienciación social de la importancia de los contenidos de la prensa.
\end{abstract}

\section{Palabras clave}

Investigación; periodismo; prensa; diarios; México.

\section{Abstract}

The study of the mass media allows know the profile of a society and their topics that all days are published in the pages of the newspapers. The contents reflected in their pages are the most current contemporary history and give a specific vision of this context. We mirada crítica ", en Revista Mediterránea de comunicación, 1, pp. 141-157. Recuperado el día de mes de año (añadir enlace) 
arrive to those topics with the analysis of content of the newspapers and in the case of Mexico this type of investigations are very few. In this article there is carried out a detailed study that confirms this affirmation and is investigated in the questions that do this type of study is not an appellant for the investigators. This article is based on the search of the bibliographical references that support the hypothesis and the methodology of the analysis of case in Colima State (Mexico). The most singular characteristics are: there isn't actual and adapted bibliography, sources hemerográficas worthy and from the point of view of the contents published in press, there isn't enough investment and interest for the topics related to the Journalism and in general with the sciences of the communication. The article proposes also the need of the investment in investigation, their later diffusion and the social assimilation of the importance of the press's contents.

\section{Key Words}

Research; journalism media; daily press; Mexico.

Sumario: 1. Introducción. 2. Metodología. 3. Resultados. 4. Reflexiones finales. 5. Bibliografía. 6. Notas.

Summary: 1. Introduction. 2. Methodology. 3. Results. 4. Final thoughts. 5. Bibliography. 6. Notes.

\section{Introducción}

\section{1. Formulación de objetivos}

La investigación científica en el área del Periodismo no tiene fronteras y cada vez son más los trabajos relativos a las Ciencias de la Información y de la Comunicación que ven la luz en cualquier parte del mundo. Los resultados son difundidos a través de las revistas especializadas impresas y digitales en función de su capacidad y colaboran en la aportación de referencias bibliográficas para nuevas propuestas metodológicas, epistemológicas y analíticas y en definitiva a trabajos académicos más especializados y ramificados que ayudan a entender la complejidad del fenómeno periodístico.

Esta obviedad refleja claramente el buen hacer de los trabajos académicos cuyas dificultades de realización quedan eclipsadas en los mismos artículos o simplemente se integran en la parte del quehacer y las rutinas metodológicas a las que se resta importancia hasta el punto de no mencionarse en la mayoría de las investigaciones científicas. En general, los investigadores no hablamos de las dificultades con las que nos encontramos cuando llevamos a cabo nuestros trabajos como si hablar de ello sólo restara credibilidad a nuestros estudios.

El propósito de este artículo que se presenta es precisamente dejar patente cuáles son los principales obstáculos de la investigación sobre Periodismo en México cuando se trata de emplear las técnicas del análisis de contenido y cuando este estudio se lleva a cabo en provincias. La finalidad de esta propuesta es despojar al investigador de la coraza que en este país y en este campo de estudio desvirtúa los resultados prácticos, conocer la realidad de un campo de estudio que tienen más de desarrollo práctico que de reflexión teórica, llevar a cabo una reflexión de estas dificultades compartidas por algunos investigadores que entre líneas las ponen de manifiesto en 
sus artículos y en conclusión, reflexionar sobre esta realidad y sus consecuencias en el ámbito de la investigación académica sobre Periodismo en México.

La búsqueda de las dificultades de investigaciones en el proceso metodológico en relación al análisis de contenido en el caso del Periodismo llevan a la propuesta de los siguientes objetivos: en primer lugar, la necesidad de describir el panorama sobre la investigación sobre Periodismo en México y en concreto con el empleo de la técnica del análisis de contenido; en segundo lugar, la localización de los problemas que obstaculizan la investigación, lo que implicará la definición del problema y justificará la importancia de la investigación que se presenta en tanto que muestra una realidad que todo investigador debe tener en cuenta previa a la propuesta de estudios que impliquen el uso de estos métodos en dicho país; finalmente y en tercer lugar, la propuesta de mejora para la superación de los obstáculos con los que se encuentran los investigadores.

Resulta necesario conocer la realidad que contextualiza el hecho que se va a investigar en este artículo con objeto de tener un acercamiento al tema de estudio. Esta aproximación implica la localización de publicaciones y artículos que tengan como tema principal las dificultades sobre la investigación en relación al Periodismo en México, así como de los artículos y publicaciones, que aunque lo traten como tema secundario, mencionen este aspecto como determinante en la investigación que se ha llevado a cabo, bien antes o bien después, de la realización del estudio propiamente dicho.

En este sentido, y siguiendo las pautas antes descritas, la necesidad de describir el panorama actual de la investigación sobre Periodismo en México implica la mención de una serie de autores que reflejan esta situación y respaldan la necesidad de reflexionar sobre las dificultades y la propuesta de mejoras para una investigación de rigor empleando la técnica del análisis de contenido cuando se trate de llevar a cabo estudios sobre medios de comunicación.

\section{2. Antecedentes y situación actual}

Los estudios en relación a los medios de comunicación mexicanos publicados por editoriales del país no son muy numerosos [1] y los existentes están unidos a propuestas políticas e intereses económicos. La referencia está orientada al estudio de los medios de comunicación en sí mismos, a sus contenidos y a la realidad que difunden con lo que dicen de manera abierta y con lo que omiten. En este sentido se han detectado varios autores que ponen de manifiesto esta realidad no sólo en el ámbito mexicano, sino en la totalidad de la investigación latinoamericana.

La investigadora Marisol Velarde Tovar $(2006,4)$ concreta que los inicios de los estudios latinoamericanos de Ciencias de la Comunicación tienen su origen en los años setenta y que suceden de manera paralela a la inclusión de propuestas gubernamentales que "se bifurcaban entre la denuncia o apoyo institucional". Añade más adelante que "México es un claro ejemplo: hasta la séptima década del siglo veinte, en nuestro país la generación de información dependía completamente de las grandes agencias informativas extranjeras; pero con la creación de agencias informativas nacionales- el mensaje fue más parcial, ya que el Estado controlaba la información reforzando con ello el carácter monolítico de los medios de comunicación".

Dentro de este campo de estudio que son los medios de comunicación, interesa conocer cómo fue el desarrollo y cuál es la situación actual del relativo a la prensa. Recordemos que la denuncia que se quiere hacer en estas páginas entra en el campo del periodismo escrito ya que es la herramienta directa del análisis de contenido, que es la estrategia metodológica que con más dificultades, como se va a demostrar en este artículo, se desarrolla desde el ámbito de la investigación académica. La razón por la que es importante investigar sobre periodismo y más en 
concreto hacerlo con el análisis de las páginas de los periódicos es un tema que está sobradamente justificado, pero en el caso de México, resulta de especial interés por la ausencia de estudios en este sector.

Sobre la investigación en relación a los medios de comunicación en México, Rosalba Cruz Soto (2000, 421) manifiesta la necesidad de investigar sobre periodismo y de cómo los trabajos publicados no son actuales y afrontan temas especializados sectoriales. Se puede interpretar que la investigadora propone de manera indirecta la necesidad de estudiar de una manera más amplia y completa y abarcando muchas más áreas los contenidos que se publican en los diarios del país:

"La investigación histórica en México no concede gran atención a la prensa. Hasta ahora son escasas las obras y poco el interés hacia esta materia. Se cuentan por miles los impresos de este tipo editados en el país que no han sido investigados (...). Lo que más se ha estudiado, sobre todo son artículos de revistas especializadas, es la prensa política y literaria. Hasta la década de los setenta aparecieron otros estudios que abordaban otros más especializados como la mujer en el periodismo, la prensa obrera y la legislación de imprenta. Todo ello se relaciona con los intereses de los mismos autores, pero sin duda también en el momento en el que se escribieron: los años setenta, cuando se vivieron aperturas en distintos campos sociales".

La crítica a la ausencia de trabajos que analicen el periodismo en México también se ha detectado por otros autores que han investigado sobre el fenómeno de la comunicación en Centroamérica y Sudamérica, es el caso de Karin Bohmann $(1989,15)$. El autor además va más allá y estima que la prensa mexicana ha tenido un gran desarrollo y sin embargo, los investigadores extranjeros no le han prestado interés, es decir, no sólo critica la falta de trabajos llevados a cabo por los mismos mexicanos -cuyos motivos pueden estar más o menos justificados por motivos políticos, de presión, falta de interés, etc.,- sino que pone de manifiesto que los académicos de otros países, tampoco le hayan demostrado interés.

"En América Latina, México pertenece a los países que disponen de una red de medios de comunicación relativamente amplia y tecnológicamente avanzada. No obstante, la investigación internacional en el terreno de la comunicación le ha prestado hasta la fecha poca atención. De igual manera, los numerosos trabajos de investigación realizados en torno al sistema político y económico de México no han tomado en cuenta el papel que desempeñan los medios de comunicación, lo cual no deja de sorprender en virtud de su importancia en el proceso de desarrollo social".

Ya se ha descrito la realidad en el ámbito de la investigación sobre comunicación en México y en general en Latinoamérica, se ha detallado también lo relativo a la prensa, y finalmente falta añadir esa descripción del panorama del tema que nos ocupa en relación al análisis de contenido. Dentro de este campo de estudio que son los medios de comunicación y en concreto el periodismo escrito, los relacionados con el análisis de contenido no tienen una gran representatividad. El análisis de contenido implica un sistema de trabajo minucioso y que requiere paciencia. No es sin embargo un método complejo y la inmensa mayoría del trabajo que se presentará será nuevo y las conclusiones suelen servir de referencia a nuevas investigaciones.

Tal vez por esa razón sea por la que el análisis de contenido resulta interesante. Carlos Águila Franco $(1990,261)$ explica de una manera muy concreta la importancia de esta metodología de estudio en el contexto de los medios de comunicación: "El análisis de información ha coadyuvado al enriquecimiento del periodismo, y es de gran utilidad para los propios periodistas, para los funcionarios, para los políticos y para todos aquellos que deseen saber lo que a través de los mensajes no se dice, y encontrar la intencionalidad y el verdadero sentido de lo que se exprese". 
Finalmente la contextualización de este panorama sobre el estudio del análisis de contenido sobre periodismo en México debe ser entendida dentro de un contexto más amplio. El problema que se va a tratar en las siguientes páginas es la ausencia de trabajos sobre análisis de contenido en medios impresos mexicanos y, en consecuencia, de referencias bibliográficas y hemerográficas. Tiene un origen cuyas raíces se asientan en la base de la cultura y el desarrollo cultural de las políticas de este ámbito.

No se trata de un problema que afecta en exclusiva a la conservación de libros y periódicos, sino también a la difusión de contenidos y de investigaciones. La ausencia de bibliografía es sólo la punta del iceberg de un problema más complejo: falta de interés cultural y/o político por la investigación en relación a la prensa y en general a los medios de comunicación. El investigador Rodrigo Centeno $(2009,2)$ ponía de relieve estas deficiencias en relación a la investigación en México:

"Sin embargo, independientemente de las tendencias políticas, hoy México está claramente rezagado en innovación y ha faltado una visión integral que impulse al país en esta materia. Gran parte de la innovación proviene del gobierno ( $46 \%$ en México vs. $29 \%$ en la OCDE). Asimismo, hemos destinado pocos recursos a esta área: México es el país en la OCDE que menor inversión realiza en investigación y desarrollo (I\&D); en el año 2007 destinó 0,5\% del PIB y \$57 dólares per cápita, vs. 2,26\% del PIB y \$694 dólares per cápita en el promedio de la OCDE”.

Las afirmaciones de Centeno son muy posteriores a las desarrolladas por otro autor que describe con una de cal y otra de arena la situación de la investigación en relación a los medios de comunicación. Héctor Gómez Vargas (2006, 11-13) afirma que la década de los 80 fue "intensa" para los estudios de comunicación en América Latina y en México, que en este periodo "se vivieron momentos importantes y decisivos" en la conformación del campo académico de la comunicación en México. "Los estudios de comunicación lo hacen para dar cuenta, predominantemente, del quehacer de los medios, simplificando en mucho una creciente realidad social y cultural, cada vez más compleja" que tuvo la pretensión de unir la cultura a la comunicación.

Y añade más adelante que este quehacer se llevó a cabo con "un débil trabajo teórico y conceptual de manera campal, con deficiencias metodológicas, así como una esquizofrenia epistemológica, la sucesión de modas y recorridos teóricos sin fin”. Finaliza esta crítica a los métodos empleados que la situación comenzó a cambiar cuando los investigadores asumieron la realidad de esta situación y comenzaron a "re-organizar" todo lo investigado e "intentaron hacer un esfuerzo para planificar un mínimo de orden de lo que fue su aparición y desarrollo".

En este repaso de la situación actual de la investigación en México, después de hablar de la falta de estudios en relación a los medios de comunicación, incluso en general de las Ciencias de la Comunicación en toda la producción latinoamericana, y más en concreto de la prensa o del periodismo escrito, pasando por la metodología del análisis de contenido, se debe sumar la circunstancia de que las fuentes bibliográficas suelen ser escasas y caducas. Así lo afirma el investigador Gustavo A. León Duarte $(2010,74-75)$ en un artículo sobre la Escuela Latinoamericana de Comunicación (ELACOM) con respecto a las revistas y el ámbito propio de la investigación en comunicación:

"Entre los problemas advertidos, se destacan la insuficiencia de los recursos teóricosmetodológicos necesarios para estudiar los fenómenos sociales asociados con las tecnologías o la urbanización contemporánea, y la obstinada pretensión por buscar instrumentos cognitivos para construir objetos comunicativos de carácter híbrido y multidisciplinares". 


\section{3. Hipótesis de estudio y justificación}

La situación actual descrita en el epígrafe anterior sirve de base para la propuesta de hipótesis que consiste en la demostración de que la metodología del análisis de contenido en el ámbito de estudios de los medios de comunicación y en concreto de la prensa apenas ha sido estudiado en México. Para dar respuesta a esta propuesta habrá que conocer cuáles son los problemas que impiden que se pueda llevar a cabo investigaciones de estas características.

La importancia de llevar a cabo una escueta aunque profunda investigación de este tema no es otra que la de dejar patente que, aunque hay muchas investigación en torno a los medios de comunicación desde otros fundamentos teóricos, y también desde otras perspectivas prácticas, el análisis de contenido no es un método válido porque la herramienta que lo hace posible no está a la mano de los investigadores. El análisis de contenido en prensa implica la localización, conservación y manipulación de los periódicos, y el problema radica en que estos no se conservan en los fondos de las bibliotecas, por lo tanto el acceso del investigador no puede tener lugar a excepción del esfuerzo de adquirirlos por su cuenta.

Dejando patente esta realidad se pretende que desde las universidades y los centros de investigación promuevan la necesidad de contar con estos fondos como base para el desarrollo de la investigación sobre medios de comunicación y en segunda instancia de la sociedad, ya que es a través de los contenidos que difunden los medios como desde diferentes ciencias como son las Ciencias de la Comunicación, la Antropología, la Sociología, pueden conocer rasgos esenciales de la sociedad. Como respaldo a esta afirmación quisiera cita a Luis Jesús Galindo Cáceres $(2008,118)$ cuando dice

"Las llamadas Ciencias de la Comunicación, a partir de la denominación sociológica crítica de las industrias culturales, ha desarrollado dentro del propio pensamiento crítico, una propuesta sobre la cultura que poco a poco se ha convertido en el centro del discurso académico sobre le tema, con diversos puntos de vista, pero con una consistencia que llama la atención sobre la importancia determinante de los medios de difusión para comprender la cultura, esa figura del siglo XIX, hoy en día".

¿Por qué es importante difundir esta realidad? Si se admite que no hay suficientes estudios en relación a los medios impresos empleando el análisis de contenido, herramienta fundamental como método de trabajo del investigador académico, se podrá comenzar a dar pasos hacia la realización de estudios en este ámbito que ayudará al desarrollo cultural en el ámbito de México porque permitirá conocer más el perfil de la sociedad. Así mismo afectará positivamente a las relaciones académicas con otros países en los que sí se están llevando a cabo este tipo de investigaciones posibilitando los estudios comparados y, en definitiva, la mejora de los trabajos académicos relacionados con los medios de comunicación.

\section{Metodología}

No se trata sólo de demostrar en este texto que la investigación de los medios de comunicación con la técnica del análisis de contenido escasea, sino de la importancia del empleo de estos recursos en las investigaciones sobre Periodismo en México. Con este fin se detallan a continuación los problemas que se han localizado en relación al tema de estudio y acorde a esta situación la propuesta de una metodología de estudio que demuestre o deseche la hipótesis de trabajo. 


\section{1. Localización del problema}

El problema fundamental que se trata de localizar es la evidencia de los principales obstáculos con los que se encuentra el investigador que desea llevar a cabo un estudio sobre periodismo en concreto periodismo impreso. En esta línea, la jefa del Departamento de Estudios de la Comunicación Social de la Universidad de Guadalajara (México), María Elena Hernández Ramírez (2008, 22-23) resulta crítica con la situación de la investigación sobre Periodismo en México:

"Es claro que hasta ahora el periodismo no se ha analizado suficientemente como objeto de estudio complejo, y que en la investigación mexicana no se ha construido o reconocido tratamiento teórico-metodológicos que den cuenta de la multidimensionalidad de estos fenómenos; o al menos no han sido aplicados con consistencia en el mundo del periodismo mexicano contemporáneo. (...) Entre lo que falta, sobresale la necesidad de discutir académicamente de la conceptualización que hacemos en la investigación sobre periodismo, y propiciar el reconocimiento del subcampo de 'estudios sobre periodismo', con la teorización que esto exige".

La ausencia de estudios relacionados con los medios de comunicación en los estados del país es una realidad. Hernández añade a su análisis la necesidad de impulsar estos temas en el país mexicano del mismo modo que ya se está realizando en otros países, y pone como ejemplo a Estados Unidos, Canadá, Alemania, y más recientemente, Francia, Brasil y Argentina. Pero la investigadora lo que destaca de la situación en la que se encuentra la investigación académica en México son las debilidades de una profesión que todavía no ha madurado en todos los sentidos, tanto en la parte profesional como en la académica y formativa. Hernández $(2008,23)$ concreta al respecto que:

"Quizás el desinterés o la poca claridad de los investigadores mexicanos para analizar los fenómenos periodísticos tengan alguna relación con la falta de consolidación de la profesión periodística en el país; con la fragilidad o la ambigüedad en la definición de las ofertas para la formación de periodistas; con la inmadurez social en materia de legislación de la comunicación; con las características de la estructura de propiedad de los medios informativos, o con todo a la vez".

A esta situación se le añade las dificultades que se pueda encontrar si el estudio que se quiere llevar a cabo emplea la técnica del análisis de contenido. Hernández considera que el estudio de los fenómenos periodísticos contemporáneos mediante el análisis de contenido "no ha constituido hasta ahora una tendencia sólida o importante dentro del campo de la investigación de la comunicación".

Quizás el problema radica en el hecho de que no se emplea el análisis de contenido porque los temas de los que se investiga en México no requieren del empleo de esta técnica. El análisis de contenido de los periódicos tiene sentido si se pretenden conocer la tendencia sobre los temas de los que se habla en los medios y que luego conformarán los temas de discusión de la opinión pública y en general de toda la población [2]; también de los géneros periodísticos empleados, los recursos tradicionales empleados en prensa y que no son comparables en las versiones digitales, etc.

Sobre cuáles son los temas de los que se investiga sobre la prensa en México cabe mencionar el estudio que desarrolla la investigadora Celia del Palacio Montiel $(2006,23)$, que considera a este respecto que "son muy pocos los trabajos que intentan vincular a los periódicos con su momento y explicar sus devenires en relación con los acontecimientos no sólo políticos, sino sociales y 
económicos". Concreta también que casi el $50 \%$ de las investigaciones que se desarrollan en México tienen que ver con estudios de cobertura informativa de algún tipo, análisis que tienen como punto de partida el terreno histórico de los acontecimientos, y que se cae con facilidad en investigaciones poco especializadas que no se concretan en el estudio de los contenidos.

Esta situación parece afectar con más virulencia a los estados que en la capital del país como diversos autores ponen de manifiesto. Celia del Palacio Montiel $(2006,24)$ dice: "Es entendible que la mayor parte de las investigaciones sobre historia de la prensa se realicen en la capital, básicamente porque ahí se encuentran los archivos y hemerotecas más completos y mejor conservados (no hablemos aquí de otros posibles factores, como los apoyos diferenciados a la investigación y la calidad también distintas de las escuelas de comunicación y de historia)".

En este sentido desde la Revista Investigación y Desarrollo (AAVV, 2000, 1) se apoya la descentralización en lo relacionado a la inversión con objeto de posibilitar el estudio y la investigación desde los estados: "El país ha concentrado la mayor parte de sus recursos humanos, materiales y de infraestructura en la zona metropolitana de la ciudad de México, por lo que las actividades científicas y tecnológicas en distintas entidades federativas se han visto rezagadas debido a dicha centralización. Por esta razón, un desarrollo equitativo refiere necesariamente al tema de la descentralización de la ciencia y la tecnología en el territorio nacional".

Del Palacio Montiel concreta en relación a las regiones que: "En las regiones, además de la falta de apoyo para la investigación, encontramos la ausencia de materiales hemerográficos, base fundamental de una investigación de este tipo. La situación de los archivos estatales y municipales en la mayor parte de los estados es lamentable, debido principalmente a que pocos lugares cuentan con los recursos económicos y humanos para la preservación adecuada de los materiales". También Rosalba Cruz Soto $(2000,1)$ afirma en relación a la investigación en prensa que "su estudio será cada vez más difícil porque los ejemplares resguardados en las hemerotecas tienden a desaparecer de los acervos, ya sea por motivos políticos, por la acción del tiempo o por actuaciones vandálicas (...)".

La ausencia de materiales bibliográficos es probablemente uno de los problemas más importantes que justificarían la ausencia de trabajos sobre periodismo empleando el análisis de contenido. En este sentido resulta de gran interés confirmar esta circunstancia. De confirmarse delataría la falta de interés por la conservación de un material impreso que refleja de manera más fiel la historia contemporánea del país, o en este caso, y como bien han descrito antes las investigadoras, la de los estados.

En este sentido, el doctor español, Daniel Narváez Torregrosa $(2007,33)$, describe de esta forma la realidad de la conservación de archivos hemerográficos, "pero cuando uno se ha curtido en los 'campos de batalla' que son los archivos regionales, las historias centralizadas dan pavor. Y dan miedo porque desde el maremagnum que es cualquier capital, la información periodística (hemerográfica es el término académico) que es con lo que se suele trabajar presenta lagunas de información y datos que sólo pueden ser contrastados in situ" (...) Los legajos de documentos parece que son muy útiles a la hora de construir barricadas y trincheras".

\section{2. Metodología empleada}

La metodología empleada para este artículo se concreta en el estudio de caso. La realidad que se describe y los problemas que se concretan describen un entorno donde la investigación sobre periodismo ha sido poco desarrollada, la poca que se ha desarrollado lo ha sido en el ámbito 
histórico, el análisis de contenido no ha sido una herramienta metodológica empleada, básicamente porque los fondos hemerográficos no existen 0 están incompletos, y fundamentalmente esto ocurre así en los estados o provincias del país.

Por esta razón se ha llevado a cabo un estudio de caso en uno de los estados más desarrollados del país, el estado de Colima [3] en México. Situado en el centro oeste es una de las regiones geográficamente más pequeñas y sin embargo de las más estables a nivel económico, político y social. Tiene una extensión de 5.500 metros cuadrados, un $0.3 \%$ del total del país. Abarca una población de unos 600.000 habitantes donde el $85 \%$ vive en zonas urbanas y el $15 \%$ en zona rural. La renta per cápita es de 5.000 dólares americanos y la tasa de analfabetismo está situada en el $8 \%$.

El estudio de caso se llevó a cabo en la ciudad de Colima (Colima-México) durante el verano de 2008 y posibilitado por una beca de investigación de la Secretaría de Relaciones Internacionales (SRE). Los estudios de caso tienen la ventaja de que suelen ser viables y facilitan la realización del trabajo: fuentes más asequibles, contraste de fuentes y datos más al alcance de la mano, localización de la información más rápida, análisis de los resultados más accesibles, etc. Pero, tienen el inconveniente de que no deja de ser un ejemplo a partir del cual el investigador invita a la generalización y siempre con la prudencia de que los resultados y conclusiones deben ser tenidos en cuenta con la limitación de que sólo se ha estudiado un caso.

En otra investigación que también empleó el estudio de caso como herramienta metodológica llevada a cabo en la ciudad de Colima en relación a las telenovelas y del cual fue responsable la profesora Ana Bertha Uribe Alvarado $(2007,135)$, la autora explica las deficiencias de su estudio en un capítulo completo que denomina 'limitaciones'. La última y cuarta de éstas hace referencia a las concreciones geográficas y dice así: "Con una rápida visión al texto, pueden saltar a la vista las siguientes [limitaciones]: (...) enfatizar sólo en las características de una región específica, en nuestro caso Colima, sin ubicarlo en un nivel de referencia nacional o global. Sería pertinente descubrir en qué medida esos casos particulares pueden ser equiparables a otros escenarios".

Uribe $(2007,136)$, haciendo uso de un tono científico y más amplio afirma más adelante que "si entendemos la ciencia y la investigación social como un proceso no acumulativo y de constante reelaboración significativa, irónicamente siempre estamos corriendo el riesgo de lo inacabado de los textos". En definitiva, la intención es lo que cuenta, y a partir de un trabajo sencillo se puede interpretar para generalizar o al menos para tenerlo en cuenta en futuras investigaciones posteriores.

El estudio de caso que aquí se presenta está basado en la realización de una investigación sobre Periodismo, en la que se quiere conocer los intereses culturales de la sociedad mexicana a través de los textos publicados en prensa El estudio se lleva a cabo en tres periódicos de tirada nacional y de gran presencia en el país: Reforma, El Universal y La Jornada. El tiempo de estudio fue de un mes de duración y se empleó de la metodología del análisis de contenido.

\section{Resultados del estudio de caso y propuesta para el desarrollo de la investigación sobre periodismo en México.}

En este capítulo se ha descrito lo que fue la experiencia de la investigación de un trabajo que se detalla a continuación y que sirve de modelo para el estudio de caso. De este modo se pretende respaldar con un ejemplo práctico basado en la experiencia los aspectos teóricos en relación al panorama actual sobre la investigación sobre Periodismo en México basado en la técnica del análisis de contenido y llevado a cabo en uno de los estados del país. Con esta experiencia y el 
apoyo de la bibliografía antes referenciada se llevarán a cabo una serie de propuestas para el desarrollo de la investigación sobre Periodismo en México para las cuales se ha contado también con el apoyo de referencias hemerográficas y bibliográficas que respalden las descritas.

\section{1. Resultados del estudio de caso}

Durante el verano de 2008 la Secretaría de Relaciones Exteriores (SRE) del Gobierno Mexicano y la Facultad de Letras y Comunicación de la Universidad de Colima (Colima) posibilitaron la investigación de un estudio sobre Periodismo. Tenía como objetivo conocer los intereses culturales de la sociedad mexicana a través de los textos publicados en prensa[4]. La misma metodología propuesta para el proyecto se tuvo que modificar en la redacción final previa a la entrega en la universidad y a la SRE por la imposibilidad en la localización del material objeto de estudio[5]. A tal fin se acudió a diversos organismos públicos -la Universidad de Colima no adquiere, y por lo tanto, no conserva, los ejemplares de periódicos de tirada nacional-: la hemeroteca del estado no conserva ejemplares actuales de diarios de tirada nacional y es Prensa de Gobierno del Estado quien sí guarda dos de los tres diarios objetos de estudio, El Universal y La Jornada. Llama la atención que sólo una dependencia oficial conservara, y de manera incompleta, la prensa publicada.

No obstante las dificultades en la localización de los contenidos precisos para llevar a cabo una investigación de esta magnitud no finalizan ahí. El acceso a la fuente fue ciertamente complejo y los ejemplares que se ofrecieron no fueron completos[6].

Así mismo cabe destacar las deficiencias en la conservación de los periódicos en Prensa del Gobierno, tanto por su ubicación, localización como modo de conservación. Todos almacenados en dos armarios y una habitación que al mismo tiempo servía de área de descanso de los empleados (con su nevera, microondas, fregadero, etc.). Guardados en enormes columnas de periódicos, donde una hoja de papel con una fecha indicaba el final de un año y el principio de otro, con severas dificultades para su manipulación que para nada indica que debe ser el modo de conservación de este tipo de documentos. Sin embargo, y aunque suene contradictorio, se debe alabar este tipo de almacenaje por el esfuerzo que supone para los responsables de su custodia en un contexto donde se evidencia que el valor dado a los archivos hemerográficos es insuficiente.

El último de los diarios que se trabajó fue Reforma, que siendo uno de los más representativos a nivel nacional, no tiene difusión en este estado. Este periódico sí que tiene, a diferencia de los otros dos, la posibilidad de ser consultado íntegramente a través de la página Web previo pago. Este sistema de consulta de las páginas en versión pdf posibilitó que todos los días pudieran ser analizados.

Finalmente, se acusó la antigüedad de los libros de consulta en las bibliotecas de la universidad, siendo pocos los ejemplares cuyas ediciones fueran posteriores al año 2000. Se echó en falta las publicaciones de autores mexicanos. De ahí que los capítulos de la investigación no se vean enriquecidos con citas de estudios anteriores publicados en México, de temas mexicanos y por autores mexicanos. [7].

Este apartado de estudio de caso de la investigación en México concluye que: 
1. A la hora de investigar a nivel hemerográfico sobre Periodismo en México, no se conservan los ejemplares de periódicos de difusión nacional y estatal, en algún caso porque ni siquiera se compran por la biblioteca o archivo específico o porque ni tan siquiera llegan a distribuirse en la zona.

2. Esto denota poco interés por la investigación sobre prensa en México desde los lugares donde es propio adquirir y conservar publicaciones diarias relacionadas.

3. Denota también poco interés por los agentes sociales de que se estudien los contenidos de los periódicos.

4. No significa que la falta de interés lo sea de los investigadores, que no tienen acceso a los periódicos como fuente de información libre y gratuita.

5. Así mismo, a la hora de buscar información bibliográfica sobre Periodismo y medios de comunicación se comprueba que los libros y revistas especializadas editados y difundidos son pocos y desfasados al menos así lo demuestra el estudio de caso en el estado de Colima.

6. En consecuencia se puede deducir que hay poco interés por la posterior difusión de estudios relacionados con el Periodismo.

\subsection{Propuestas para el desarrollo de la investigación sobre Periodismo en México}

La percepción subjetiva de los autores hasta este momento y los resultados del estudio de caso que concluyen en las dificultades reales de investigar sobre periodismo y medios de comunicación en México, tienen como consecuencia que: al ser difícil investigar, cuando se logra, se guardan con celo los resultados. Así lo expone el profesor Daniel Narváez Torregrosa $(2007,34)$ cuando afirma en relación a la investigación en México que "este ejercicio de reconstrucción no se puede hacer en solitario, ni como ermitaño celoso de que le arrebaten sus conocimientos. Es necesario sistematizar, intercambiar y dialogar, ejercicios que en definitiva ayudará a escribir y recuperar el patrimonio de una manera más rápida, con diversidad de opiniones y libre de cualquier discurso maniqueo (...)".

A este respecto quisiera añadir una cita de Arturo Merayo $(2007,6)$ en el prólogo del libro sobre la investigación de la comunicación en Iberoamérica cuando dice que "una de las principales carencias en el ámbito del estudio de la comunicación ha sido el escaso intercambio establecido entre los investigadores". El volumen analiza ampliamente el estado de la investigación tanto en España como en Iberoamérica desde el trabajo realizado de manera conjunta por instituciones diversas. El libro en general ilustra una imagen optimista sobre la investigación académica en aquellos artículos en los que se pone de manifiesto, sin embargo es evidente que los proyectos que se describen están centrados principalmente en estudios bibliográficos en temas políticos, económicos y sociales, fundamentalmente, y poco o nada sobre el análisis de contenido y el trabajo de campo desde la perspectiva del Periodismo. Desde este punto de vista también es adecuada la afirmación de Merayo $(2007,7)$ cuando dice que "curiosa paradoja ésta: no hay suficiente comunicación en las Ciencias de la Comunicación".

Este problema en relación a la escasa difusión de conocimiento es compartido por la población académica y tiene llamativos ejemplos en el caso de la investigación mexicana. Según las fuentes consultadas y que se han citado en este artículo se trata de un problema relacionado con la inadecuada inversión en investigación y no con un problema de base cultural en relación a los beneficios de compartir conocimientos. Como ejemplo este texto de Xavier Gamboa Villafranca (1981, 6-19) donde habla de la labor desarrollada por el Centro de Investigaciones de Quintana 
Roo, A.C. (CIQRO). A pesar de la antigüedad que tiene el artículo su contenido sirve para ilustrar, a modo de ejemplo -casi histórico- una situación que en apariencia sigue siendo actual:

"Los organismos de investigación existentes en el país afrontan serios problemas, internos y externos. (...) A pesar de la confianza depositada por las autoridades de investidura, los órganos de autoridad del CIQRO han impedido que ésta se desarrolle en toda su potencialidad. La incapacidad académica, el individualismo, la posición política y la perspectiva ideológica, se entrelazan, para conformar un conjunto de factores que distorsionan la actividad de la Dirección General del Centro(...)".

Gamboa acusa al centro de "despilfarrar los recursos públicos" y concreta que algunas de las anomalías que se han producido son: "la construcción de la imagen pública para consumo externo", "la progresiva autocensura, tanto para la presentación de resultados parciales o finales de investigación, cuanto en la estrategia y práctica de recolección de datos de campo", y añade que "el centro se ha convertido en un desproporcionado, complejo y costoso aparato administrativo. La situación ha llegado al extremo de que, para septiembre de este año [1981], sólo 12 personas -de un total de más de cuarenta- se dedicaban realmente, de tiempo completo, a investigar. Esto es lo que explica que, a pesar del enorme presupuesto del año 1979, y del correspondiente al de 1980, sólo un estudio había sido terminado" [8].

Casi 30 años después de aquellas declaraciones, no faltan referentes que critiquen la mala gestión de las inversiones que se destina a investigación, la falta de recursos reales, así como la complejidad burocrática para solicitar realización de proyectos. Otro ejemplo lo suscribe las declaraciones de Alberto Efendy Maldonado Gómez de la Torre (2001, 109): "A pesar del significativo crecimiento de la investigación de la comunicación durante los ochenta y los noventa, la investigación teórica sigue siendo un campo muy restringido, donde trabajaban pocos investigadores y con fuertes problemas de circulación en el medio académico".

La circulación o difusión de los trabajos no es el único problema, son muchos otros los que encuentran los investigadores antes de llegar a este punto. De este modo, Rodrigo Centeno $(2009,2-7)$ realiza una reflexión en relación a la innovación extrapolables a los relacionaos con la investigación. El autor considera que el problema principal de la innovación no es analizar las razones por las que no se invierte en esta materia e incluso en investigación, sino analizar las barreras que imposibilitan que esto cambie. Concreta que básicamente los problemas surgen como consecuencia de la ausencia de un organismo nacional que gestione todas las operaciones, facilitando el trabajo y homogeneizando sistemáticas de trabajo que agilicen los trámites burocráticos que obstaculizan la creación y suponen el fracaso de la gestión.

"La razón inmediata detrás de la falta de competitividad de México, en lo que respecta a asignación de gasto en innovación, radica en que los incentivos no se encuentran correctamente coordinados entre todos los agentes que participan en el proceso; es decir, al no haber un plan nacional consolidado en materia de innovación el resultado es que los pocos recursos con los que se cuenta se gastan de manera poco eficiente".

La propuesta del autor es un Sistema Nacional de Innovación (SIN) donde se concreten proyectos asequibles, "una visión nacional del trabajo, dotando de capacidad de gestión a la autoridad encargada". Lo cierto es que la complejidad del sistema burocrático invita a la desconfianza. El autor dice al respecto que "otra barrera a la entrada que es posible eliminar con una sola instancia es la duplicación de procesos burocráticos. Contar con una dependencia única que coordine todos los proyectos de innovación hará posible contar con procesos burocráticos consolidados, lo que reducirá tiempos y costos a los emprendedores, y fijará incentivos a la inversión privada en proyectos de innovación". Totalmente trasladable al caso de la investigación académica. 
La casi nula relación entre estados en esta materia es evidente, pero la inercia y la rutina parecen suficiente argumento como para mantener este sistema. El mismo Centeno (2009, 3-4) pone de relieve esta descoordinación cuando dice "los actores no están coordinados y no hay una autoridad con la capacidad de gestión suficiente para hacerlo posible". Y apuesta por un modelo de gestión coordinado "que permita que los incentivos se encuentren alineados de manera que se promueva la coordinación entre dependencias y niveles de gobierno". Centeno confirma esta circunstancia: "esto es un problema fundamentalmente económico expresado en la inadecuada alineación de incentivos y en las ineficiencias generadas por procesos burocráticos excesivos y mal diseñados", y añade más adelante "la falta de coordinación tiene efectos nocivos sobre los incentivos de los emprendedores mexicanos y con ello limita su disponibilidad a innovar".

La Revista Investigación y Desarrollo $(2000,1)$ realiza la siguiente propuesta que hoy en día suena a utopía, pero que no por ello merece ser olvidada: "para lograr el desarrollo científico y tecnológico en México, el gobierno tendrá que promover la descentralización de las actividades científicas y tecnológicas; aumentar la formación de recursos humanos calificados; incrementar el financiamiento; (...) promover la difusión y divulgación; integrar el sistema científico y tecnológico a los sectores social, gubernamental y empresarial; y, establecer un marco legislativo adecuado en todos los niveles de gobierno".

\section{3. Discusión de los resultados y respuesta a la hipótesis de trabajo}

Es una realidad a la que se enfrenta cualquier investigador en México que resulta complejo encontrar buenos fondos hemerográficos en las provincias del país. Llevar a cabo, por lo tanto, una investigación basada en la metodología del análisis de contenido de prensa mediadamente actual es casi un trabajo imposible -siempre con la excepción de que el investigador se compre sus propios periódicos-. La falta de archivos que conserven estos fondos, así como la publicación de trabajos que suelen emanar de estos estudios brillan por su ausencia y así lo ponen de manifiesto autores mexicanos en artículos que han publicado en diferentes revistas.

La falta de inversión en investigación académica es visible tras rastrear cuáles son los trabajos académicos publicados y las opiniones de los expertos que han estudiado el tema. No hay recursos para la adquisición de los periódicos por parte de las administraciones públicas en provincias -como se ha podido demostrar con el estudio de caso-, o no hay interés cultural por la conservación de los recursos hemerográficos, y en definitiva quien se resiente es el desarrollo de la investigación. Es el pez que se muerde la cola: el profesor o investigador no cuenta con facilidad en el acceso a las fuentes, de modo que prefiere llevar a cabo otros estudios que sí le permiten este acceso, así queda fuera el estudio de temas sobre periodismo empleando la metodología del análisis de contenido y la actualidad que es contada a través de los medios de comunicación escritos no es analizada, es decir, el referente escrito más cercano a la historia contemporánea no recibe interés por parte de los investigadores.

El objetivo principal de este texto ha sido demostrar la falta de estudios sobre periodismo y en concreto los relativos al análisis de contenido de prensa y este trabajo ha dejado al descubierto la necesidad de invertir en investigación académica sobre Periodismo en México (en concreto la prensa actual) uniendo las voces de algunos autores que se han localizado y que critican esta situación. La falta de inversión y desvío de recursos ponen de manifiesto una realidad que convierte en compleja y dificultosa la investigación doctoral y posdoctoral en este país. La metodología que se han empleado en esta demostración han sido doble: por una parte, las referencias bibliográficas en relación al tema y por otra el estudio de caso de una investigación sobre Periodismo llevada a cabo en México. 
Así mismo, otro objetivo que se persigue es reflejar las propuestas para el desarrollo y la mejora de la investigación en este país, tanto las que plantean los investigadores como las que surgen como consecuencia del estudio de caso. Las propuestas pasan por la descentralización de la investigación y fomentar la inversión en provincias para que éstas puedan llevar a cabo trabajos interesantes, y al mismo tiempo un solo órgano administrativo que coordine todo el trabajo, y dé difusión a las investigaciones realizadas, como funciones básicas y prioritarias.

Los inversores empresariales, políticos y culturales no ven con interés la investigación relacionada con el Periodismo y en concreto el estudio de los medios impresos, y este problema es con toda probabilidad uno de los más complejos y que más frena el desarrollo de este campo en México. Si la investigación no revierte de manera útil en la sociedad, o no se le ve la manera en la que puede hacerlo, los investigadores tampoco le prestan atención porque no sienten que se verán valorados por el esfuerzo realizado.

Evidentemente, estas carencias las sufre el investigador mexicano y el investigador extranjero con las repercusiones negativas que implica en el segundo caso. Por un lado, la iniciativa y posterior decepción del que decide investigar en México y, por otro lado, pone freno a la posible visita de investigadores extranjeros. El mexicano no tiene de otra que enfrentarse a esta situación si quiere o tiene que desarrollar su faceta investigadora, y al final, adapta su trabajo, objetivos y metodología al material que le es accesible. De este modo investiga determinando sus contenidos al acceso a las fuentes y, generalizando, siempre son los mismos temas los que son objeto de estudio, frente a áreas que se dejan de investigar por desidia ante el inoperante acceso a los recursos y las fuentes. Como consecuencia el investigador se muestra celoso de compartir aquel material que tanto le costó localizar e incluso las conclusiones que se derivaron de éste.

En diciembre de 2000, la Revista Investigación y Desarrollo $(2000,1)$ concreta que la investigación en México pasa por una adecuada inversión y cambio de mentalidad a nivel cultural: "La gestión de la ciencia y la tecnología deberá entenderse como una inversión y no como un subsidio, si se desea que el país pueda competir en un mundo globalizado".

Hoy en día investigar en México existen dificultades que obstaculizan el trabajo y que tienen su origen en aspectos culturales. Difícil porque no se trata sólo de aumentar la inversión en infraestructura, o recursos humanos o tecnología, ya que como ha quedado demostrado en el artículo cuando se han tenido los recursos para trabajar adecuadamente no se han sabido aprovechar. Otros autores achacan las dificultades organizativas, burocráticas y de gestión a la falta de conciencia de la importancia de los medios de comunicación como agentes sociales que también interfieren en la historia contemporánea de un país, también a los recursos que posibiliten la conservación de archivos adecuados, etc.

Las dificultades de la investigación en México tienen difícil solución porque son varias y porque todas interfieren entre sí. De modo que deberían solucionarse de manera conjunta y paulatina para ver los resultados adecuados. Según los autores podría deberse a una falta de inversión en recursos para la investigación o a la falta de organización y gestión de los ya existentes, pasando por la necesidad de homogeneizar los sistemas de gestión y de centralizarlos. En definitiva, se percibe el mensaje de que la investigación en Periodismo no es importante y se pone de relieve el silencio de los entes públicos a las propuestas de los investigadores y académicos, eludiendo así la responsabilidad que no es sólo una cuestión económica. 


\section{Reflexiones finales}

La hipótesis que se planteaba al inicio del artículo en relación a la demostración de que apenas se ha estudiado la prensa en México con la técnica del análisis de contenido se cumple, como así lo confirman las referencias bibliográficas y el estudio de caso. Este técnica metodológica no es válida porque los investigadores no pueden acceder a las fuentes primarias, en este caso a los periódicos impresos, porque no se conservan adecuadamente o no se conservan en absoluto porque se tiran o porque no se adquieren en los puntos de referencia para los investigadores, es decir, las bibliotecas, fondos bibliográficos y fondos hemerográficos. Asumida esta realidad se puede empezar a hablar de las deficiencias en la estructura económica, política y social que impiden o que posibilitarían que esta situación cambiara, ya que la realización de estudios en el ámbito del Periodismo ayudará al desarrollo cultural en México porque permitirá conocer más el perfil de la sociedad actual.

Evidentemente, quien logra algún tipo de inversión para investigar es celoso de compartir los contenidos que tanto trabajo y tiempo le ha llevado. Este fenómeno que se produce a nivel individual de los mismos investigadores se reproduce a gran escala en los estados, donde el resentimiento a la centralización de archivos en la capital del país, desemboca en una descentralizada y mal organizada gestión de recursos para investigación. En definitiva, hay poca producción, pocas publicaciones, poca difusión.

Investigar en provincias en México sobre Periodismo y los medios de comunicación, desde la metodología del análisis de contenido (con base hemerográfica actual), es desaconsejable por todos los argumentos expuestos. Si desde las altas esferas políticas del país hay un interés por mejorar la investigación en México ésta pasa por considerar el estudio de los medios de comunicación y del Periodismo tan importante como cualquier otra ciencia.

\section{Bibliografía}

ÁGUILA FRANCO, C (1990): ‘El periodismo mexicano hoy'. En AA.VV.: El periodismo mexicano hoy. México, UNAM.

AAVV. (2000): "Pide la Rednacecyt que la investigación en México sea prioridad nacional". En Revista Investigación y Desarrollo. Periodismo de Ciencia y Tecnología. http://www.invdes.com.mx/anteriores/Diciembre2000/htm/red.html. Última consulta el 1 de junio de 2010.

BOHMANN, K. (1989): Medios de comunicación y sistemas informativos en México. México, Alianza Editorial Mexicana.

CENTENO, R. (2009): "Propuesta de cambio. Innovación en México: un reto de coordinación y apropiación". En CIDAC. Centro de Investigaciones para el Desarrollo. http://www.cidac.org/vnm/pdf/propuestas/innovacionenmexico.html. Última consulta el 1 de junio de 2010.

CRUZ SOTO, R. (2000): "El periódico, un documento historiográfico". En Del Palacio Montiel, C. (2006): La prensa como objeto de estudio. Panorama de las formas de hacer historia de la prensa de México. Comunicación y Sociedad, 5, Pp. 421. 
DEL PALACIO MONTIEL, C. (2006): "La prensa como objeto de estudio. Panorama de las formas de hacer historia de la prensa de México". Comunicación y Sociedad, 5, Pp.11-34.

GALINDO CÁCERES, L.J. (2008): Comunicología y Estudios culturales. "Encuentro entre la comunicación y la cultura visto desde el inicio del siglo XXl", Estudios sobre las culturas contemporáneas. Colima (México). Junio, pp. 113-131.

GAMBOA VILLAFRANCA, X. (1981): "EI CIQRO: distorsiones en un centro de investigación". Revista Catorcenal Independiente. 1, 1. pp. 6-19.

GÓMEZ VARGAS, H. (2006): "Figuras del pensar. Los estudios sobre el Consumo Cultural en América latina y la organización del campo académico de la comunicación en México, notas sobre su trayectoria, mapas y reorganización", Estudios sobre las culturas contemporáneas. Colima (México). Junio, pp. 10-43.

HERNÁNDEZ RAMÍREZ, M.E. (2008): "La investigación sobre periodismo en México". Revista Universidad de Guadalajara, 22, 7. http://www.cge.udg.mx/revistaudg/rug22/rug22dossier7.html. Última consulta el 1 de junio de 2010.

JURADO MARTÍN, M. (2009): Cultura y Espectáculos en la prensa de México. Estudio de temas y géneros en los periódicos: Reforma, El Universal y La Jornada. Alicante, ECU.

LARA KLAHR, M. (2005): Diarismo. Cultura e industria del periodismo impreso en México y el mundo. México, Edita Análisis, Ediciones y Cultura.

LEÓN DUARTE, G. A. (2010). "Estrategias, posiciones y prácticas científicas en la enseñanza y la investigación de la comunicación en América latina", en Miguel Hernández Communication Journal, 1. Páginas 53-77. Universidad Miguel Hernández, UMH (Elche-Alicante). http://mhcj.es/2010/02/01/gustavo-leon-duarte . Última consulta el 1 de junio de 2010.

MALDONADO GÓMEZ DE LA TORRE, A.E. (2001): Comunicación. Campo y objetivo de estudio. Perspectivas reflexivas latinoamericanas. Colima (México), Universidad de Colima.

MERAYO, A. (2007): "Presentación. La investigación de la comunicación en Iberoamérica: historia, estado actual y nuevos retos". Sphera Pública. Revista de Ciencias Sociales y de la comunicación. Número especial 2007. Pp. 7-15.

NARVÁEZ TORREGROSA, D. (2007): "La azarosa aventura de historial el cine". Panorama. Revista de la Universidad Autónoma de Baja California Sur. 53. Pp 31-34.

PÉREZ ESPINO, E. (1991): "Los motivos de Televisa. El proyecto cultural de Xeq Canal 9. México, Instituto de investigaciones sociales". México. Universidad Nacional Autónoma de México.

URIBE ALVARADO, A.B. (2007): "Un encuentro para celebrar. El estudio de las telenovelas", Estudios sobre las culturas contemporáneas. Colima (México). Junio, pp. 135-136.

VELARDE TOVAR, M. (2006): "Los retos de las teorías de la comunicación en el siglo XXI". Revista Digital Universitaria. México, junio, número 6. http://www.revista.unam.mx /vol.7/num6/art51/int51.htm . Última consulta el 1 de junio de 2010.

YTUARTE NÚÑEZ, C. (2000) "Principales rasgos culturales del periodismo y la prensa mexicana". En DEL PALACIO MONTIEL, C. (comp.) Historia de la Prensa en Iberoamérica. México, Alianza del Texto Universitario. 


\section{Notas}

[1] La referencia generaliza a partir de la investigación llevada a cabo a través del rastreo de las fuentes bibliográficas de la biblioteca de la Facultad de Humanidades de la Universidad de Colima (México), de la biblioteca de Periodismo de la Universidad de Colima (México) y de los artículos accesibles en red en revistas especializadas que se citan como bibliografía en el presente texto, así como las bases de datos y la red de revistas Científicas de América Latina y el caribe, España y Portugal (Redalyc) y Dialnet.

[2] También se podría usar la técnica del análisis del discurso y en tal caso los acceso de las páginas Web de los periódicos servirían de herramienta, aunque en este caso se descarta todo lo relativo al uso de las unidades cuantitativas medidas en centímetros o módulos, así como el resto de virtudes que emanan del estudio basado en el análisis de contenido.

[3] Los datos han sido extraídos de la web monográficos.com. http://www.monografias.com/trabajos15/estados-mexico/estados-mexico.shtml. Última consulta el 28/05/10.

[4] El proyecto de investigación 'Prensa escrita: géneros periodísticos y estilo temático de los periódicos mexicanos: Reforma, El Universal y La Jornada' tuvo por objeto el estudio de los géneros periodísticos con los que trabajan los medios de comunicación mexicanos y el estilo temático en su sección cultural. (...) Los objetivos fueron conocer los periódicos mexicanos de tirada y difusión nacional, analizar el contenido de un número limitado de estos periódicos (Reforma, El Universal y La Jornada), durante el mes de junio en la sección de Cultura. Se pretendió también conocer a los académicos y profesionales que en México hayan publicado estudios similares y conocer sus textos. La investigación fue publicada con la siguiente referencia: JURADO MARTÍN, M. (2009): Cultura y Espectáculos en la prensa de México. Estudio de temas y géneros en los periódicos: Reforma, El Universal y La Jornada. Alicante, ECU.

[5] El mes propuesto a priori fue febrero, para hacerlo coincidir con el mismo que ya se tenía realizado de diarios españoles y de cara a la futura comparación de los resultados. Pero fueron muy numerosos los problemas hallados, y el principal de todos fue la imposibilidad de tener acceso a las versiones impresas o incluso las versiones en formato pdf de los diarios. La opción más eficaz fue que la investigadora los comprara ya que la Universidad de Colima no los adquiere. En su área de prensa se conservan y guardan los últimos meses sólo de diarios del estado de Colima, y la biblioteca de Periodismo no tiene hemeroteca de los ejemplares nacionales, ni locales.

[6] El acceso a estos documentos fue dilatado hasta tal punto que casi llega a suponer el cambio del tema de la investigación. Los responsables de los Servicios de Prensa de Gobierno del Estado de Colima no contestaron a la petición de la Universidad de Colima acerca del permiso que la investigadora requería para tener acceso a sus ejemplares de archivo. Finalmente, por otras vías de carácter personal se obtuvo este permiso verbal y no oficial. Durante este dilatado proceso y ante el miedo de no poder trabajar con los periódicos, la investigadora los fue adquiriendo a partir de la segunda quincena del mes. Finalmente con la primera quincena de El Universal y La Jornada pudo trabajar consultándolos en las instalaciones gubernamentales. No obstante alguno de estos diarios había desaparecido del archivo.

[7] Al hablar de bibliotecas de la universidad se está descartando los libros que son propiedad del profesorado y/o aquellos que de manera habitual se conservan en sus despachos y no son de libre acceso.

[8] Solicitar disculpas al lector de este texto por la antigüedad de la referencia de 1981, que denota no la falta de interés de la investigadora que firma, sino la ausencia de textos publicados accesibles posteriores para ser citados. 